asHoka

UNIVERSITY

ASHOKA UNIVERSITY ECONOMICS

DISCUSSION PAPER NO. 36

\title{
Dominant Strategy Implementation in a Large Population Public Goods Game
}

September 2020

Ratul Lahkar, Ashoka University

Saptarshi Mukherjee, Indian Institute of Technology, Delhi 


\title{
Dominant Strategy Implementation in a Large Population Public Goods Game
}

\author{
Ratul Lahkar* Saptarshi Mukherjee ${ }^{\dagger}$
}

August 22, 2020

\begin{abstract}
We consider implementation of the efficient state in a large population public goods game. Agents are divided into a finite set of types. The planner asks agents to report types, which generates a reported type distribution. Based on reported types and distribution, the planner calculates the efficient strategy level and a Pigouvian transfer for each type of agent. We show that this direct mechanism satisfies incentive compatibility in strictly dominant strategies, strong budget balance and ex-post individual rationality.
\end{abstract}

Keywords: Public Goods; Externalities; Pigouvian Pricing; VCG Mechanism.

JEL classification: C72; D62; D82.

* Department of Economics, Ashoka University, Rajiv Gandhi Education City, Sonipat, Haryana 131029, India, email: ratul.lahkar@ashoka.edu.in.

${ }^{\dagger}$ (corresponding author) Department of Humanities and Social Sciences, Indian Institute of Technology Delhi, Hauz Khas, New Delhi, India, email: saptarshi.isi@gmail.com 


\section{Introduction}

Implementation of the efficient outcome in a public goods provision model is an enduring problem in microeconomic theory. Since agents do not internalize positive externalities in such a situation, the equilibrium outcome falls short of the efficient outcome. Pigouvian pricing is the most wellknown solution to such a problem. This, however, requires the social planner to possess considerable information about agents' individual characteristics. Mechanism design approaches circumvent this problem but raises other issues. The classical VCG mechanism for public goods induces truthful revelation of type as a weakly dominant strategy but does not satisfy budget balance, at least in a strong sense (Moulin [8]). ${ }^{1}$ An alternative is the AGV mechanism that induces truthful revelation as a Bayesian Nash equilibrium (Arrow [1], d'Aspremont and Gérard-Varet [3]). But this mechanism does not satisfy ex-post individual rationality and also assumes that the type distribution is common knowledge.

This paper addresses these classical issues by adopting a large population approach. A continuum of agents of finitely many types play a public goods game in which the benefit depends upon the aggregate strategy level and the cost upon individual strategy. Following the intuition of Pigouvian pricing and the classical VCG mechanism, we introduce a direct mechanism in which the planner calculates optimal strategies on the basis of reported types and offers a transfer to agents that equals the externality created by the agent minus a lumpsum amount. This mechanism, therefore, makes no assumption about the planner's knowledge of the type distribution. The fact that each agent is of measure zero then induces truthful revelation, and not just as a weakly dominant but as a strictly dominant strategy. In addition, we also obtain strong budget balance and ex-post individual rationality. Of course, in reality, no society has a continuum of agents. Therefore, like the model of a perfectly competitive market, our results are relevant only when the number of agents are sufficiently large that they take aggregate social variables as given.

Lahkar and Mukherjee [6] applied evolutionary implementation (Sandholm [9]) to achieve efficiency in a large population public goods model. ${ }^{2}$ The present paper applies a more conventional mechanism design approach in which the planner creates incentives for truthful revelation. The possibility of reconciling efficiency with incentive compatibility in large economies was first noted by Hurwicz [5] and extended to incorporate public goods by Hammond [4]. We go beyond possibility results and explicitly characterize the mechanism and its properties. Makowski and Ostroy [7] extend VCG mechanisms to large economies with public goods and establish dominant strategy implementation along with budget balance and individual rationality. They, however, only consider quasi-linear preferences while we do not impose any such restriction. These papers are also in a general equilibrium framework while ours is a partial equilibrium model (more in line with the classical VCG setting in a large economy). As mentioned earlier though, our results are stronger

\footnotetext{
${ }^{1}$ Strong budget balance means the total transfer made by the planner should be zero. VCG mechanisms at best ensure a negative total transfer, which leaves a surplus with the planner.

${ }^{2}$ In evolutionary implementation, the planner imposes an externality price to induce convergence to the efficient state. There is no reliance on truthful revelation in such a model.
} 
than in conventional VCG mechanisms in the sense that we also obtain strong budget balance in which the planner is left with neither surplus nor deficit.

Section 2 presents the model and discusses Pigouvian pricing under the assumption that the planner knows the type distribution. In Section 3, we weaken the informational assumption and characterize the mechanism that implements efficiency in dominant strategies. Section 4 concludes.

\section{Pigouvian Pricing}

We consider a society of a continuum of agents of mass 1 . Thus, each agent is of measure zero. The society is divided into a finite set of populations $\mathcal{P}=\{1,2, \cdots, n\}$, which we interpret as types. We denote the type distribution as $m=\left(m_{1}, m_{2}, \cdots, m_{n}\right)$, with $\sum_{p \in \mathcal{P}} m_{p}=1$. Thus, $m_{p}$ denotes the mass or proportion of agents of type $p \in \mathcal{P}$. Each agent in the society has the strategy set $\mathcal{S}=[0, \infty)$. We denote by the finite positive measure $\mu_{p}$ the distribution of strategies in population $p$, with $\mu_{p}(A) \in \mathcal{S}$ denoting the mass of type-p agents playing strategies in $A \subseteq \mathcal{S} .^{3}$. Equivalently, $\mu_{p}$ is the state of population $p$. If all agents in a population $p$ play the same strategy $x$, then that is a monomorphic population state which we denote as $m_{p} \delta_{x}$.

A social state is then $\mu=\left(\mu_{1}, \mu_{2}, \cdots, \mu_{n}\right)$. To introduce a large population public goods game, we define the aggregate strategy level

$$
A(\mu)=\sum_{p \in \mathcal{P}} \int_{\mathcal{S}} x \mu_{p}(d x)=\int_{\mathcal{S}} x \sum_{p \in \mathcal{P}} \mu_{p}(d x) .
$$

Note that $A(\mu) \in[0, \infty) .{ }^{4}$ The payoff of a type-p agent who plays strategy $x$ in the public goods game is then

$$
F_{x, p}(\mu)=v(A(\mu))-c_{p}(x)
$$

where $v:[0, \infty) \rightarrow \mathbf{R}_{+}$is the common benefit function and $c_{p}: \mathcal{S} \rightarrow \mathbf{R}_{+}$are the type specific individual cost functions. We assume that all such functions are strictly increasing, smooth and satisfy $v(0)=c_{p}(0)=0$ for all $p \in \mathcal{P}$. Further, $v$ is concave and $c_{p}$ is strictly convex for all $p \in \mathcal{P}$. The public goods character of (2) arises because the benefit is due to aggregate strategy whereas cost is due to individual strategy. This game also constitutes an aggregative game as the payoffs depend only upon individual strategy and the aggregate strategy (Corchón [2]). We describe this game concisely as $F$.

Since every agent is of measure zero, the action of a single agent has no effect on aggregate variables. Hence, the benefit part of (2) is independent of a single agent's strategy. Therefore, the Nash equilibrium and, in fact, the dominant strategy equilibrium of $F$ is $\mu^{N}=\left\{m_{1} \delta_{0}, \cdots, m_{n} \delta_{0}\right\}$. All agents in all populations play the 0 strategy at this equilibrium.

\footnotetext{
${ }^{3}$ Note that $\mu_{p}(\mathcal{S})=m_{p}$ for all $p \in \mathcal{P}$.

${ }^{4}$ Depending upon context, strategies may represent effort or monetary contributions to a public project. The aggregate strategy would then be aggregate effort or aggregate monetary contribution.
} 
Using (2), we calculate the aggregate payoff at a state $\mu$ in our public goods game $F$ as

$$
\begin{aligned}
& \sum_{p \in \mathcal{P}} \int_{\mathcal{S}} F_{x, p}(\mu) \mu_{p}(d x) \\
= & \sum_{p \in \mathcal{P}} \int_{\mathcal{S}}\left(v(A(\mu))-c_{p}(x)\right) \mu_{p}(d x) \\
= & v(A(\mu))-\sum_{p \in \mathcal{P}} \int_{\mathcal{S}} c_{p}(x) \mu_{p}(d x) .
\end{aligned}
$$

An efficient state of $F$ is a social state $\mu^{*}$ that maximizes (3). To identify such a state, we introduce the function

$$
v\left(\sum_{q \in \mathcal{P}} m_{q} \alpha_{q}\right)-\sum_{p \in \mathcal{P}} m_{p} c_{p}\left(\alpha_{p}\right) .
$$

We characterize the efficient state in the following proposition. The proof follows from Lemma 3.3 and Proposition 3.4 in Lahkar and Mukherjee [6].

Proposition 2.1 The public goods game $F$ defined by (2) has a unique efficient state (up to a difference of a measure zero of agents) $\mu^{*}=\left(m_{1} \delta_{\alpha_{1}^{*}}, \cdots, m_{n} \delta_{\alpha_{n}^{*}}\right)$, where $\left(\alpha_{1}^{*}, \cdots, \alpha_{n}^{*}\right) \in \prod_{p=1}^{n}(0, \infty)$ is the unique maximizer of (4).

The result follows because, as shown in Lahkar and Mukherjee [6], (3) is concave in $\mu$ with a unique maximizer. Further, the maximizer must be in monomorphic population states. At such states, (3) equals (4) if $x=\alpha_{p}$ for all type $-p$ agents. The assumptions about $v$ and $c_{p}$ imply that $\left(\alpha_{1}^{*}, \cdots, \alpha_{n}^{*}\right) \in \prod_{p=1}^{n}(0, \infty)$. If we denote

$$
\alpha^{*}=\sum_{p \in \mathcal{P}} m_{p} \alpha_{p}^{*}
$$

then (4) implies that each $\alpha_{p}^{*}$ defined in Proposition 2.1 is characterized by

$$
v^{\prime}\left(\alpha^{*}\right)=c_{p}^{\prime}\left(\alpha_{p}^{*}\right)
$$

where $\alpha^{*}$ is as defined in (5). Notice that $\alpha^{*}=A\left(\mu^{*}\right)>0$. Thus, the aggregate strategy at the efficient state is strictly greater than that at the Nash equilibrium, which is a characteristic of all public good models.

We introduce a planner who seeks to implement the efficient state. Suppose the planner knows the functions $\left\{v, c_{1}, c_{2}, \cdots, c_{n}\right\}$ and, more importantly, the type distribution $m$. In that case, we argue that the planner can implement the efficient state $\mu^{*}$ using standard Pigouvian pricing. To do so, the planner uses (4) and (5) to calculate $\alpha^{*}=A\left(\mu^{*}\right)$. The planner then announces $v\left(\alpha^{*}\right)+x v^{\prime}\left(\alpha^{*}\right)$ as the benefit for an agent who plays strategy $x$. This is independent of an agent's type so that the planner has no need to know the type to make this announcement. A type- $p$ 
agent who plays strategy $x$ then subtracts her private cost $c_{p}(x)$ thereby generating the payoff

$$
\begin{aligned}
& F_{x, p}\left(\mu^{*}\right)+x v^{\prime}\left(A\left(\mu^{*}\right)\right) \\
= & v\left(\alpha^{*}\right)-c_{p}(x)+x v^{\prime}\left(\alpha^{*}\right)
\end{aligned}
$$

for such an agent.

Intuitively, $x v^{\prime}\left(\alpha^{*}\right)$ is the subsidy given by the planner to an agent playing $x$ to induce her to internalize the positive externality she creates at the efficient state $\mu^{*} .{ }^{5}$ This is of course standard Pigouvian pricing with $x v^{\prime}\left(\alpha^{*}\right)$ being the Pigouvian transfer. Further, (7) is a simple individual maximization problem. If we assume that agents are standard payoff maximizers, then a type $-p$ agent will clearly choose $x=\alpha_{p}^{*}$ as characterized in (6). Thus, Pigouvian pricing implements the efficient state in our public goods model.

\section{Dominant Strategy Implementation}

In applying Pigouvian pricing, the planner needs to know the type distribution $m$ but not the type of individual agents. But suppose the planner doesn't even know the type distribution. This is a typical mechanism design problem, which we analyze using the standard approach of a direct mechanism. The planner asks every agents to report her type from $\mathcal{P}=\{1,2, \cdots, n\}$ and then assigns a strategy and transfer to every agent based on reported type. We call this direct mechanism $\phi$. The planner would like to design $\phi$ such that for any type distribution, the efficient state is implemented in equilibrium. Throughout, we continue to assume that the planner knows the benefit function $v$ and the possible cost functions $\left\{c_{1}, c_{2}, \cdots, c_{n}\right\}$.

Agents are free to report their types truthfully or falsely. Suppose that the distribution generated by the reported types is $\tilde{m}=\left\{\tilde{m}_{1}, \tilde{m}_{2}, \cdots, \tilde{m}_{n}\right\}$. Thus, $\tilde{m}_{p}$ is the proportion of agents who report their type to be $p$ or, equivalently, their cost function to be $c_{p}$. It is possible that $\tilde{m}_{p} \neq m_{p}$. If an agent announces type to be $q \in\{1, \ldots, n\}$, then the planner assigns strategy $\tilde{\alpha}_{q}^{*}$ and the transfer $\tilde{\alpha}_{q}^{*} v^{\prime}\left(\tilde{\alpha}^{*}\right)-\tilde{\alpha}^{*} v^{\prime}\left(\tilde{\alpha}^{*}\right)$ to that agent, where $\tilde{\alpha}^{*}=\sum_{p \in \mathcal{P}} \tilde{m}_{p} \tilde{\alpha}_{p}^{*}$ and $\left(\tilde{\alpha}_{1}^{*}, \cdots, \tilde{\alpha}_{n}^{*}\right)$ is the maximizer of (4) when we replace $m$ with $\tilde{m} .{ }^{6}$ Since the planner knows the function $v$ and $\left\{c_{1}, c_{2}, \cdots, c_{n}\right\}$, these calculations are feasible.

Thus, the payoff of a type- $p$ agent in $\phi$ who announces type to be $q$ and when the reported type distribution is $\tilde{m}$ is

$$
\phi_{p}(q ; \tilde{m})=v\left(\tilde{\alpha}^{*}\right)+\left(\tilde{\alpha}_{q}^{*} v^{\prime}\left(\tilde{\alpha}^{*}\right)-\tilde{\alpha}^{*} v^{\prime}\left(\tilde{\alpha}^{*}\right)\right)-c_{p}\left(\tilde{\alpha}_{q}^{*}\right)
$$

\footnotetext{
${ }^{5}$ More generally, $x v^{\prime}(A(\mu))$ is the positive externality imposed by agents who uses strategy $x$ at state $\mu$ on the society. It is the marginal impact on the payoff (3) of other agents when the mass of strategy $x$ users increases. This interpretation of positive externalities can be formalized using the calculations in Appendix A.1.1 in Lahkar and Mukherjee [6]. It is this positive externality that causes the aggregate strategy at the efficient state to be greater than that at the Nash equilibrium in our model.

${ }^{6}$ Formally, $(q, \tilde{m}) \mapsto\left(\tilde{\alpha}_{q}^{*}, \tilde{\alpha}_{q}^{*} v^{\prime}\left(\tilde{\alpha}^{*}\right)-\tilde{\alpha}^{*} v^{\prime}\left(\tilde{\alpha}^{*}\right)\right)$ is the output function of the mechanism $\phi$. Reported type $q$ and reported type distribution $\tilde{m}$ leads to strategy and transfer allotment $\left(\tilde{\alpha}_{q}^{*}, \tilde{\alpha}_{q}^{*} v^{\prime}\left(\tilde{\alpha}^{*}\right)-\tilde{\alpha}^{*} v^{\prime}\left(\tilde{\alpha}^{*}\right)\right)$.
} 
Once again, because agents are of measure zero, reports by a single agent does not affect $\tilde{m}$ and, therefore, also does not affect the aggregate variable $\tilde{\alpha}^{*}$. Hence, the terms $v\left(\tilde{\alpha}^{*}\right)$ and $\tilde{\alpha}^{*} v^{\prime}\left(\tilde{\alpha}^{*}\right)$ are strategically irrelevant in (8). Nevertheless, they will play an important role in the main result of this paper where we show that $\phi$ possesses three important desirable characteristics in a mechanism-incentive compatibility, individual rationality and budget balance.

Proposition 3.1 For any type distribution $m$, the mechanism $\phi$ defined by (8) implements the efficient state $\mu^{*}$ in the public goods game $F$ defined by (2). Further, $\phi$ satisfies incentive compatibility in strictly dominant strategies, ex-post individual rationality and budget balance.

Proof. Budget balance is the most obvious. It requires that the total transfer made by the planner be zero. Since $\tilde{\alpha}^{*}=\sum_{p \in \mathcal{P}} \tilde{m}_{p} \tilde{\alpha}_{p}^{*}$, we can calculate the total transfer in $\phi$ to be

$$
\sum_{q \in \mathcal{P}} \tilde{m}_{q}\left(\tilde{\alpha}_{q}^{*} v^{\prime}\left(\tilde{\alpha}^{*}\right)-\tilde{\alpha}^{*} v^{\prime}\left(\tilde{\alpha}^{*}\right)\right)=0
$$

Hence, $\phi$ satisfies budget balance.

Incentive compatibility requires that in the equilibrium of $\phi$, every agent reveals her type truthfully. Since $\tilde{\alpha}^{*} v^{\prime}\left(\tilde{\alpha}^{*}\right)$ and $v\left(\tilde{\alpha}^{*}\right)$ are strategically irrelevant, we can reinterpret our problem in (8) to be one where a type- $p$ agent chooses $\tilde{\alpha}_{q}^{*}$ to maximize $\tilde{\alpha}_{q}^{*} v^{\prime}\left(\tilde{\alpha}^{*}\right)-c_{p}\left(\tilde{\alpha}_{q}^{*}\right)$. But once we replace $m$ with $\tilde{m},(6)$ implies that the unique solution to this problem is $\alpha_{p}^{*}$.

Therefore, $\phi$ ensures truthful revelation. Moreover, truthful revelation is a dominant strategy because it happens irrespective of the reported type distribution $\tilde{m}$. In fact, dominance is strict because $\tilde{\alpha}_{p}^{*}$ is the unique maximizer of (8).

Ex-post individual rationality requires that in equilibrium, no agent obtains a negative payoff. In equilibrium, due to truthful revelation, $\tilde{\alpha}_{p}^{*}=\alpha_{p}^{*}$ and $\tilde{m}=m$. Therefore, using (8), we can write the equilibrium payoff of a type $-p$ agent in $\phi$ as

$$
\begin{aligned}
\phi_{p}(p, m) & =v\left(\alpha^{*}\right)+\left(\alpha_{p}^{*} v^{\prime}\left(\alpha^{*}\right)-\alpha^{*} v^{\prime}\left(\alpha^{*}\right)\right)-c_{p}\left(\alpha_{p}^{*}\right) \\
& =\left[v\left(\alpha^{*}\right)-\alpha^{*} v^{\prime}\left(\alpha^{*}\right)\right]+\left[\alpha_{p}^{*} v^{\prime}\left(\alpha^{*}\right)-c_{p}\left(\alpha_{p}^{*}\right)\right],
\end{aligned}
$$

We need to show that $\phi_{p}(p, m) \geq 0$. The second term in $(10), \alpha_{p}^{*} v^{\prime}\left(\alpha^{*}\right)-c_{p}\left(\alpha_{p}^{*}\right)>0$. This is because by $(6), \alpha_{p}^{*}$ is the unique maximizer of $x v^{\prime}\left(\alpha^{*}\right)-c_{p}(x)$ in $\mathcal{S}$. The properties of $v$ and $c_{p}$ imply that this maximum value is strictly positive. The first term, $v\left(\alpha^{*}\right)-\alpha^{*} v^{\prime}\left(\alpha^{*}\right) \geq 0$, with the inequality being strict if $v$ is strictly concave. This is because by concavity of $v, \frac{v(\alpha)}{\alpha} \geq v^{\prime}(\alpha)$ for all $\alpha \in[0, \infty)$. Hence, $\phi(p ; m)>0$.

The intuition behind the mechanism $\phi$ is the same as Pigouvian pricing but with the true distribution $m$ replaced by the reported distribution $\tilde{m}$. The large population characteristic of our model then ensures incentive compatibility. No agent can individually affect $\tilde{\alpha}^{*}$ through their report. Hence, the problem in (8) reduces to maximizing $\tilde{\alpha}_{q}^{*} v^{\prime}\left(\tilde{\alpha}^{*}\right)-c_{p}\left(\tilde{\alpha}_{q}^{*}\right)$ for a type-p agent 
which is solved at $\tilde{\alpha}_{p}^{*}$. Every agent, therefore, reveals type truthfully so that $\tilde{m}=m$. Each agent gets allotted the efficient strategy level $\alpha_{p}^{*}$, which implements the efficient state $\mu^{*}$ (Propositon 2.1).

Since individual agents cannot affect $\tilde{\alpha}^{*}$, the term $\tilde{\alpha}^{*} v^{\prime}\left(\tilde{\alpha}^{*}\right)$ becomes a lumpsum. This ensures

budget balance. Without it, budget balance is obviously not satisfied as every agent would receive a positive transfer $\tilde{\alpha}_{p}^{*} v^{\prime}\left(\tilde{\alpha}^{*}\right)$. We note that budget balance is satisfied in the strong sense that total transfer is zero rather than being just negative. Individual rationality ensures that no coercion is required to induce participation in the mechanism. It is significant that this is satisfied in the expost sense instead of an expected ex-ante sense. The actual equilibrium payoff is strictly positive.

\section{Conclusion}

We have, therefore, shown that our mechanism $\phi$ satisfies incentive compatibility, budget balance and individual rationality in the strongest possible sense. Hence, when applied to the large population scenario, this mechanism combines the virtues of the classical finite-player VCG and AGV mechanisms for implementing efficiency in public goods provision. As discussed in the Introduction, neither of these mechanisms satisfy all three properties, at least in the strong sense of Proposition 3.1 .

The key factor that drives our results is that individual reports do not influence the reported type distribution thereby making truthful revelation incentive compatible. It is interesting to compare this with Hammond [4], which characterizes the class of incentive compatible mechanisms in large economies using the concept of decentralizability. Decentralizable mechanisms are the ones where an individual agent is unable to affect the distribution of reported types in a large economy. We have used a similar line of argument and actually characterized such a mechanism. Makowski and Ostroy [7] show that for individual rationality to hold in large economy VCG mechanisms, individuals need to fully internalize externalities. In our model also, agents internalize externalities and individual rationality holds. But we do not require preferences to be quasi-linear.

\section{References}

[1] Arrow K (1979) The Property Rights Doctrine and Demand Revelation Under Incomplete Information. in Economics and Human Welfare, ed. by M. Boskin. New York: Academic Press.

[2] Corchón L (1994) Comparative statics for aggregative games the strong concavity case. Math. Soc. Sci. 28:151-165.

[3] d'Aspremont C, Gérard-Varet L-A (1979) Incentives and incomplete information. J. Public Econ. 11: 25-45.

[4] Hammond P (1979) Straightforward Individual Incentive Compatibility in Large Economies. Review of Economic Studies 46: 263-282 
[5] Hurwicz L (1972) On Informationally Decentralized Systems, ch. 14, pp. 297-336 of McGuire, C. B. and Radner, R. (eds.) Decision and Organization (North-Holland).

[6] Lahkar R, Mukherjee S. (2019) Evolutionary Implementation in a Public Goods Game. J. Econ. Theory 181:423-460.

[7] Makowski L, Ostroy J (1992) Vickrey-Clarke-Groves mechanisms in continuum economies: Characterization and existence. J. Math. Econ, 21:1-35.

[8] Moulin H (2009) Almost budget-balanced VCG mechanisms to assign multiple objects. J. Econ. Theory 144:96-119.

[9] Sandholm WH (2002) Evolutionary Implementation and Congestion Pricing. Rev. Econ. Stud 69:667-689. 